\title{
Problems identified in antenatal screening in Bope Poddala, Sri Lanka
}

\author{
Darshana I. L. A. N., Nanayakkara N. P., Fernando N. S., Mahendra R., Sandya L. M., De Silva P. V. \\ Department of Community Medicine, Faculty of Medicine, University of Ruhuna, Sri Lanka
}

Email address:

ilandare@gmail.com ( Darshana I.L.A.N.),npnmtxx@yahoo.com (Nanayakkara N. P.)

\section{To cite this article:}

Darshana I. L. A. N., Nanayakkara N. P., Fernando N. S., Mahendra R., Sandya L. M., De Silva P. V.. Problems Identified in Antenatal Screening in Bope Poddala, Sri Lanka. European Journal of Preventive Medicine. Vol. 2, No. 6, 2014, pp. 105-109. doi: $10.11648 /$ j.ejpm.20140206.15

\begin{abstract}
Introduction: This study was carried out to analyze the patterns and identify some co-relates of Hemoglobin levels and basic investigations (VDRL, UFR, Grouping \& Rh) performed at booking visit during pregnancy. Methods: All pregnant mothers who attended the routine antenatal clinic for their booking visit in Bope Poddala MOH area from April 2013 to April 2014 were selected as the sample. Data were extracted from the records available in the Department of Community Medicine, Faculty of Medicine, University of Ruhuna, Sri Lanka. Results Total number of mothers in the sample was 289 . Of them, $7.3 \%$ were teenage mothers while those aged between $19-35 \mathrm{yrs}$ and above $35 \mathrm{yrs}$ constituted $82.0 \%$ and $10.7 \%$ respectively. The ethnic distribution of pregnant women is as follows; Sinhala 68.2\% (197), Muslim 30.4\% (88) and Tamil 1.4\% (4). Their respective Hb levels were $12.15 \mathrm{~g} / \mathrm{dl}, 11.94 \mathrm{~g} / \mathrm{dl}$ and $10.82 \mathrm{~g} / \mathrm{dl}$. The overall mean Hb level of pregnant mothers is $12.07 \mathrm{~g} / \mathrm{dl}(\mathrm{SD}=1.48)$. The mean $\mathrm{Hb}$ levels of teenage mothers, mothers aged between 19-35yrs and mothers above 35yrs were 11.65 (SD=1.29) g/dl, $12.07 \mathrm{~g} / \mathrm{dl}(\mathrm{SD}=1.43)$ and $12.3(\mathrm{SD}=1.91) \mathrm{g} / \mathrm{dl}$ respectively. Accordingly, 22.8\% among first trimester pregnant mothers were anemic. There was no association between age group ( $\mathrm{P}$ value 0.438$)$, ethnicity ( $\mathrm{P}$ value 0.230$)$ and parity $(\mathrm{P}$ value 0.291$)$ of pregnant mothers with their Hb levels. Only $0.3 \%$ has a weakly reactive VDRL results and no one had a positive test. Out of total pregnant mothers $8.7 \%$ had pyuria and $4.5 \%$ had trace urine albumin levels at booking visit. Out of total pregnant mothers $91.7 \%$ was $\mathrm{Rh}$ positive and $8.3 \%$ was $\mathrm{Rh}$ negative. Conclusion This study shows that nearly quarter of first trimester pregnant mothers who presented at their booking visit to antenatal clinic were anemic. A considerable percentage had pyuria which is compatible with the global prevalence. Nutritional interventions for pre-pregnant women and educating pregnant women on prevention of urinary tract infections are recommended.
\end{abstract}

Keywords: Pregnancy, Basic Investigations, Booking Visit, Anemia

\section{Introduction}

Pregnancy is an important life event not only for the women but also for the whole society. It can become critical for both mother and fetus with co-morbidities. Early identification of those co-morbidities are helpful to minimize the complications related to pregnancy.

In an antenatal clinic Hemoglobin level $(\mathrm{Hb})$, Urine Full Report (UFR), Blood grouping and Rh, VDRL are routinely done in order to identify certain risk conditions. However those test are not freely available in field antenatal clinics in Sri Lanka. The Department of Community Medicine, Faculty of Medicine University of Ruhuna., has been providing the service of the above routine investigations for more than two decades as a part of the service provisions to the Bope Poddala health unit area.

Anemia in pregnancy, defined as hemoglobin concentration less than $11 \mathrm{~g} / \mathrm{dl}$, affects more than 56 million women globally, $2 / 3$ of them being from Asia. ${ }^{1}$ Anemia during pregnancy can cause both maternal and fetal complications. Some of maternal complications are less exercise tolerability, lack of tolerability in postpartum hemorrhage, heart failure, etc. ${ }^{2}$ Fetal complications are low birth weight ,preterm delivery , etc. ${ }^{2}$ Further, anemia is responsible for $20 \%$ of maternal deaths in the third world countries. ${ }^{3}$ Previously, one research has been done to determine the prevalence of anemia during pregnancy in Anuradhapura district, Sri Lanka \& it revealed prevalence of anemia in the district of Anuradhapura was less than $50 \%$ of the estimated prevalence for Sri Lanka. ${ }^{4}$ Among Sri Lankan pregnant mothers $56.1 \%$ are screened for anemia using 
hemoglobin levels at antenatal clinic visit and by them $8.2 \%$ are found to have anemia. Among this anemic mothers $7.5 \%$ have moderate anemia $(7.0-9.9 \mathrm{~g} / \mathrm{dl})$ and $0.7 \%$ have severe anemia $(<7.0 \mathrm{~g} / \mathrm{dl}){ }^{5}$

Many factors determine the $\mathrm{Hb}$ levels of pregnant women such as parity ${ }^{6}$, age ${ }^{7}$ and other health conditions. Early identification and management of low levels of $\mathrm{Hb}$ in pregnancy could prevent adverse outcomes such as postpartum hemorrhages, low birth weight and preterm deliveries etc. ${ }^{2}$ Hence it is recommended to check Hb levels of every pregnant women preferably at the first trimester.

In Sri Lanka no studies have been done to identify prevalence of urinary tract infections during pregnancy. Urinary tract infections (UTIs) during pregnancy are the commonest health problems worldwide, especially in developing countries. ${ }^{8}$ It has several adverse outcomes not only on the mother such as pyelonephritis but also on the fetus as well such as low birth weight $\&$ preterm delivery. ${ }^{2}$ In Sri Lanka still, as mentioned previously, no studies have been done to identify prevalence of UTIs during pregnancy. But a follow up study on 249 pregnant women attending the antenatal care clinic at Zagazig university hospital, Egypt in 2005 revealed that the incidence of UTIs during pregnancy was $31.3 \%{ }^{8}$ Worldwide $8 \%$ of women have asymptomatic bacteriuria and if this is untreated it may progress to UTI or even Pyelonephritis. ${ }^{2}$

Among Sri Lankan pregnant mothers, 51.2\% tested for VDRL at antenatal clinic. By them $6.7 \%$ have positive VDRL test. $^{5}$ In 2011, 25.9\% pregnant mothers in Sri Lanka were tested for blood group and Rh test at antenatal clinic. ${ }^{5}$

In this study we have selected pregnant mothers who attend the antenatal clinic because in Sri Lanka 95.9\% pregnant mothers attend at least one clinic visit during their antenatal period. ${ }^{5}$ Therefore the validity of the research is high due to high percentage of participation.

Usually in Sri Lanka, during routine antenatal clinic setup basic investigations such as Hemoglobin level, Urine for Full Report, VDRL, HIV 1 \& 2, Grouping \& Rh and Post Prandial Blood Sugar (PPBS) level are done. These services are freely provided by Department of community medicine, Faculty of Medicine Ruhuna since many years for Bope Poddala $\mathrm{MOH}$ area including 17 Public Health midwives' areas.

Bope poddala $\mathrm{MOH}$ division has a diverse population, which includes a population with different ethnicities and age groups. Still, there has been no study conducted to identify prevalence of anemia in pregnant mothers in this area. Since hemoglobin levels are routinely measured at antenatal clinic by the Department of community medicine in faculty of Medicine, Ruhuna, it was worthwhile to do a study based on these values.

This study was conducted to analyze the patterns and to identify some co-relates of hemoglobin levels and other basic investigations; VDRL, UFR, Grouping \& Rh performed at booking visit during pregnancy in Bope Poddala $\mathrm{MOH}$ area, Galle, Sri Lanka.

In Sri Lanka, under the antenatal care Hb level, UFR, Blood grouping \& Rh, VDRL and PPBS level are routinely done as an basic investigation package at booking visit in order to identify certain risk factors early and to prevent late complications that related to those risk factors. Therefore we analyzed $\mathrm{Hb}$ level, UFR, Blood grouping \& Rh and VDRL test results together although there were no direct connection between those four investigations. Hence Department of community medicine, Faculty of Medicine Ruhuna has no facilities to perform PPBS level, analysis of PPBS level was not included in the study. Although it was a limitation, available all four investigations were analyzed together to give a complete overview about antenatal screening and problem identification at booking visit during pregnancy in Bope Poddala $\mathrm{MOH}$ area, Galle, Sri Lanka.

\section{Methods}

This descriptive cross sectional study was conducted among all the pregnant mothers who attend to routine antenatal clinic for booking visit in $\mathrm{MOH}$ clinics at Bope Poddala $\mathrm{MOH}$ area between the time periods from April 2013 to April 2014. Data was extracted from the records available in the Department of Community Medicine, faculty of Medicine, Galle, Sri Lanka. Data extraction sheets were used to obtain data. All data were coded and entered in to an excel data sheet. Data cleaning and checking was done before analysis. Cross tabulation for variables was done and proportions and means were calculated where appropriate. Chi-square test was used to compare groups and to determine the statistical significance of the associations between variables.

The study was carried out after obtaining ethical clearance from the Ethical Review Committee, Faculty of Medicine, Galle.

Table 1. Socio-demographic distribution of the study sample. $(n=289)$

\begin{tabular}{lll}
\hline$"$ & Number $(\mathbf{n})$ & Percentage $(\%)$ \\
\hline Ethnicity & & \\
Sinhala & 197 & 68.2 \\
Muslim & 88 & 30.4 \\
Tamil & 4 & 1.4 \\
Age category & & \\
Teenage (<19 years) & 21 & 7.3 \\
Average (19-35 years) & 237 & 82.0 \\
Elderly ( $>35$ years) & 31 & 10.7 \\
Parity & & \\
$\mathrm{P}_{1}$ & 107 & 37.0 \\
$\mathrm{P}_{2}$ & 87 & 30.1 \\
$\mathrm{P}_{3}$ & 62 & 21.5 \\
$\mathrm{P}_{4}$ & 18 & 6.2 \\
$\mathrm{P}_{5}$ and above & 15 & 5.2 \\
\hline
\end{tabular}

\section{Results}

Two hundred and eighty nine $(n=289)$ pregnant mothers were included in the study. Among them majority were Sinhalese and belong to average age group. Nearly $2 / 3$ of mothers presented in their first or second pregnancy. Among teenage mothers as well as elderly mothers majority were Sinhalese. (Teenage mothers $\mathrm{n}=11,52.3 \%$ and Elderly mothers $n=21,67.7 \%$ ). 
The socio-demographic distribution of the study sample is shown in table 1.

The mean $\mathrm{Hb}$ levels with corresponding SD value are mentioned in Table 2 according to their ethnicity, age group and parity. Tamil, Teenage and mothers presented in the $4^{\text {th }}$ pregnancy had lowest mean $\mathrm{Hb}$ levels respectively according to their ethnicity, age group and parity. Distribution of $\mathrm{Hb}$ levels among study population is shown in Graph 1.
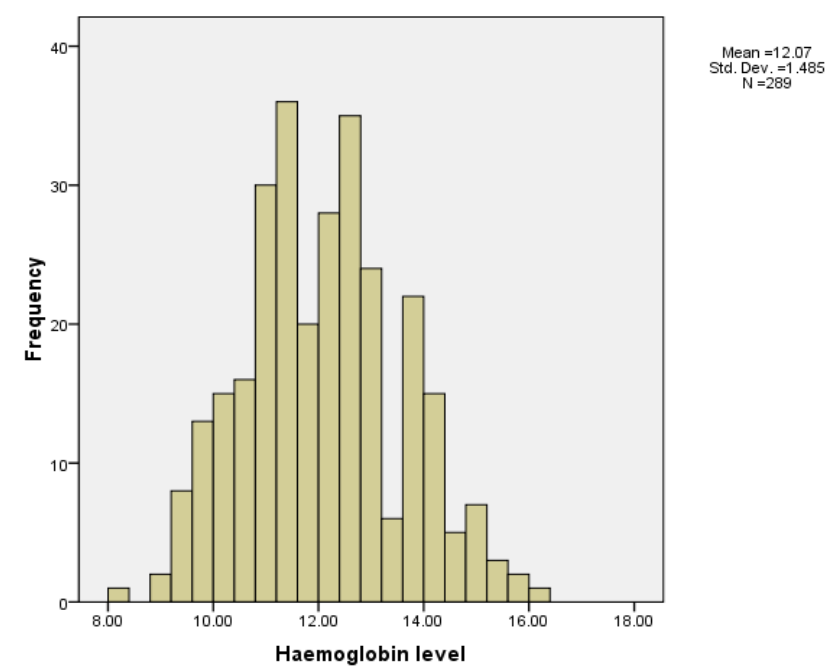

Graph 1. Distribution of Hb level among study population

Table 2. Mean Hb level vs Ethnicity, age category \& parity ( $n=289)$

\begin{tabular}{lll}
\hline & Mean Hb level & SD \\
\hline Ethnicity & & \\
Sinhala & 12.15 & 1.50 \\
Muslim & 11.94 & 1.40 \\
Tamil & 10.82 & 1.67 \\
Age category & & \\
Teenage & 11.65 & 1.29 \\
Average & 12.07 & 1.43 \\
Elderly & 12.30 & 1.91 \\
Parity & & \\
$\mathrm{P}_{1}$ & 12.12 & 1.41 \\
$\mathrm{P}_{2}$ & 12.11 & 1.59 \\
$\mathrm{P}_{3}$ & 1.48 \\
$\mathrm{P}_{4}$ & 12.15 & 1.15 \\
$\mathrm{P}_{5}$ and above & 11.35 & 1.59 \\
Total & 11.98 & 1.48 \\
\hline
\end{tabular}

Table 3. Association of anemia vs Age category, Ethnicity and Parity

\begin{tabular}{lll}
\hline Variable & Chi value & P value \\
\hline Age category & 0.601 & 0.438 \\
Ethnicity & 1.440 & 0.230 \\
Parity & 1.117 & 0.291 \\
\hline
\end{tabular}

Among study sample, when consider the parity of pregnant mothers, Tamil has highest mean parity which was 3.00 $(\mathrm{SD}=2.44)$, then Muslim 2.55(SD=1.39) \& lowest in Sinhalese $1.96(\mathrm{SD}=1.07)$. But there was no statistically significant difference between parity of pregnant mothers \& their respective $\mathrm{Hb}$ levels ( $\mathrm{P}$ value is 0.291 )

When consider the type of anemia, among the study sample, majority were not anemic (77.2\%) and only $22.8 \%$ were identified as anemic. Among them 14.5\% were mild, $8.3 \%$ were moderate \& no severe anemic. None of PHM area was not identified as endemic area for anemia \& all anemic pregnant mothers were nearly equally distributed among PHM areas.

Table 4 shows Prevalence of anemia among Ethnicities, Age categories, and Parities. Among Sinhala and Muslim pregnant mothers nearly $1 / 4^{\text {th }}$ were anemic and among Tamil pregnant mothers $3 / 4$ were anemic. But there was no statistically significant difference between ethnicity of pregnant mothers \& their respective $\mathrm{Hb}$ levels ( $\mathrm{P}$ value is 0.230 ). When consider the age category, $29.3 \%$ of elderly pregnant mothers were anemic and less than $25 \%$ of pregnant mothers were anemic among teenage and average group pregnant mothers. There was no statistically significant difference between age category of pregnant mothers and their respective $\mathrm{Hb}$ levels ( $\mathrm{P}$ value is 0.438$)$.

Table 4. Prevalence of anemia among Ethnicities, Age categories, and Parities

\begin{tabular}{lllll}
\hline & Anaemic & \multicolumn{2}{c}{ Not Anaemic } \\
\hline Ethnicity & $\mathrm{n}$ & $\%$ & $\mathrm{n}$ & $\%$ \\
Sinhala & 41 & 20.8 & 156 & 79.2 \\
Muslim & 22 & 25.0 & 66 & 75.0 \\
Tamil & 3 & 75.0 & 1 & 25.0 \\
Age category & & & & \\
Teenage & 5 & 23.8 & 16 & 76.2 \\
Average & 52 & 21.9 & 85 & 78.1 \\
Elderly & 9 & 29.3 & 22 & 70.7 \\
Parity & & & & \\
$\mathrm{P}_{1}$ & 21 & 19.6 & 86 & 81.4 \\
$\mathrm{P}_{2}$ & 19 & 21.8 & 68 & 78.6 \\
$\mathrm{P}_{3}$ & 15 & 24.1 & 47 & 75.9 \\
$\mathrm{P}_{4}$ & 6 & 33.3 & 12 & 66.6 \\
$\mathrm{P}_{5} \&>$ & 5 & 33.3 & 10 & 66.6 \\
\hline
\end{tabular}

Only $0.3 \%(n=1)$ had a weakly reactive VDRL results and no one had a positive test. Others $(n=288,99.7 \%)$ were non-reactive for the test. Out of total pregnant mothers $8.7 \%$ $(n=25)$ had pyuria. Among the sample $4.5 \%(n=13)$ had trace urine albumin levels \& albumin was nil in $95.5 \%(n=276)$ at booking visit.

Out of total pregnant mothers $91.7 \%(n=265)$ were $\mathrm{Rh}$ positive and $8.3 \%(n=24)$ were Rh negative (figure 1). Graph 2 shows distribution of blood group among the study sample.

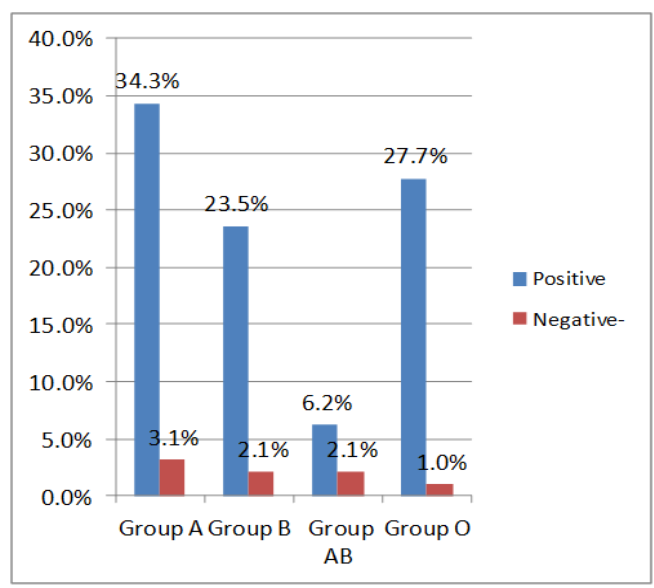

Graph 2. Blood group distribution among study sample $(n=289)$ 


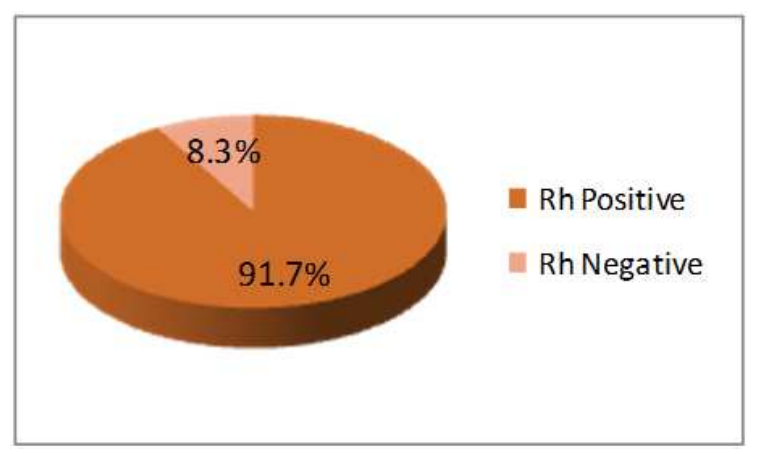

Figure 1. Prevalence of Blood group category

\section{Discussion}

Bope Poddala MOH area has a population of 70000 . It comprises of 17 PHM areas. Since1993 Bope poddala has been the field training area to study about primary health care for medical students in faculty of Medicine, University of Ruhuna. Department of community medicine assists the $\mathrm{MOH}$ of Bope poddala to carry out health programs by providing manpower and technical assistance. Although basic investigations are not supplied by $\mathrm{MOH}$ unit in Sri Lanka, pregnant mothers in Bope Poddala $\mathrm{MOH}$ unit have the privilege of receiving those facilities with help of Faculty of Medicine, University of Ruhuna. Our study aim was to analyze the patterns and identify some co-relates of Hemoglobin levels and basic investigations (VDRL, UFR and Grouping \& Rh) carried out at booking visit during pregnancy in Bope Poddala $\mathrm{MOH}$ area. This type of study has not been done in Bope Poddala $\mathrm{MOH}$ area and therefore it was worthwhile to do a study based on these investigations.

Our study shows that nearly quarter of first trimester pregnant mothers who presented at their booking visit to antenatal clinic were including mild anemia. Among Sri Lankan pregnant mothers, $7.5 \%$ are found to have moderate anemia and $0.7 \%$ have severe anemia, but mild anemia is not included in the study results. ${ }^{5}$ In our study $8.3 \%$ had moderate anemia and severe anemia was not detected. So this result is nearly compatible with the national figures. In national figures only $56.1 \%$ pregnant mothers were screened for anemia at antenatal clinic visit, but in our study all pregnant mothers in Bope Poddala $\mathrm{MOH}$ area were screened. Therefore the validity of the research is high due to high percentage of participation in our study.

A prospective study was carried out at two Medical Officer of Health areas in a district in Sri Lanka in 2010, to determine the effect of maternal $\mathrm{Hb}$ levels at booking visit on pregnancy outcome, including 817 pregnant mothers who were followed up until delivery. It showed that the prevalence of anemia ( $\mathrm{Hb}$ $<11 \mathrm{~g} / \mathrm{dl}$ ) was $7.1 \%$ (95\% CI 5.4-8.9\%). ${ }^{9}$ This study result included mild anemia too and if it compared with our study prevalence was one- third from our study prevalence.

Also there was a study done to determine the prevalence of anemia during pregnancy in Anuradhapura district and evaluate present screening methods for anemia. That study shows, in the first, second and third trimesters, prevalence of anemia was $7.6 \%, 19.7 \%$ and $19.3 \%$ respectively. ${ }^{4}$ When compare this study results with our study, prevalence of anemia during first trimester is lower than Bope Poddala $\mathrm{MOH}$ area. Although we evaluated anemia during first trimester, it is recommended the need of further evaluation of anemia during second and third trimesters because that study result showed high prevalence of anemia during second and third trimesters than first trimester.

Multiparty is a well-known cause for maternal anemia \& as well as teenage and elderly mothers are more prone to have anemia during the pregnancy. But our study shows that there was no statistically significant association between age group $\&$ parity of pregnant mothers with their $\mathrm{Hb}$ levels. A retrospective population-based cohort study was conducted using data on all singleton births $(n=290662)$ recorded in the Finnish Medical Birth Register in Finland during 2006-2010 and it revealed the prevalence of anemia during pregnancy was $2.5 \%$ among primi mothers and $2.3 \%$ among multiparous mothers. But in our study the prevalence of anemia during pregnancy was $19.6 \%$ among primi mothers and $33.3 \%$ among multiparous mothers which is very higher when comparing to that study. ${ }^{6}$

Pregnancy induces significant anatomical and physiological changes in the entire urinary tract and it facilitates the occurrence of urinary tract infections. Therefore urinary tract infections (UTIs) are relatively common in pregnancy. In Sri Lankan set up pregnant mothers are not routinely screened for asymptomatic bacteriuria. So this condition is not diagnosed commonly. But it is advisable for every pregnant woman to get a UFR and if it shows more than 10-15 pus ells in urine in the high power field a repeat UFR followed by a urine culture is mandatory. The global prevalence of asymptomatic bacteriuria is $8.0 \%$. In our study set up all pregnant mothers were screened for UTIs and a considerable percentage had pyuria, which is compatible with global prevalence. There was a follow up study on 249 pregnant women attending the antenatal care clinic at Zagazig university hospital, Egypt in 2005 revealed that the incidence of UTIs during the pregnancy was $31.3 \%{ }^{8}$ and prevalence of UTIs is nearly one quarter in our study.

Recording the blood group at booking visit will help with cross matching blood at a later date if an emergency arises. Other than that early identification of rhesus system of mother's blood group is very important to prevent complications related to 'Rhesus Isoimmunization'.In Sri Lanka, $5-7 \%$ of the population is said to have the Rh Negative blood group. But our study population, $8.3 \%$ had Rh Negative blood group which is little bit higher than national figures. ${ }^{10}$

All pregnant mothers are routinely screened for syphilis by performing VDRL test at booking visit and all clinically and / or VDRL positive cases are investigated serologically for confirmation of diagnosis as well as for other sexually transmitted diseases. Syphilis is common in many developing countries, where up to $10 \%$ of pregnant mothers may have positive serological tests. ${ }^{2}$ Among Sri Lankan pregnant mothers, $51.2 \%$ tested for VDRL at booking visit and positive test are detected by them $6.7 \%{ }^{5}$ But in our study sample no 
one was detected as VDRL positive.

At the end of the study, nutritional interventions for pre-pregnant women including health education and reassuring the receiving nutritional supplements mainly iron therapy to all pregnant mothers is recommended to minimize anemia among population. Educating pregnant women on prevention of urinary tract infection is also worthwhile.

\section{Acknowledgment}

The study was supported by all academic \& non-academic staff members of Department of Community Medicine, Faculty of MedicineUniversity of Ruhuna and staff members in Bope Poddala MOH unit.

\section{References}

[1] Goonewardene M, Shehata M, Hamad A. Anaemia in pregnancy.Best Pract Res Clin Obstet Gynaecol. 2012 Feb; 26(1):3-24.

[2] Baker P N, Kenny LC. Obstetrics by Ten teachers' $19^{\text {th }}$ edition, 2011

[3] Konar H, D.C.DUTTA's Textbook of Obstetric, $7^{\text {th }}$ edition, 2011
[4] Chathurani U, Dharshika I, Galgamuwa D, Wickramasinghe ND, Agampodi TC, Agampodi SB. Anaemia in pregnancy in the District of Anuradhapura, Sri Lanka- need for updating prevalence data\& screening strategies.Ceylon Med J. 2012 Sep;57(3):101-6.

[5] Annual report of health 2011(published by family health bureau)

[6] Räisänen S, Kancherla V, Gissler M, Kramer MR, Heinonen S. Adverse Perinatal Outcomes Associated with Moderate or Severe Maternal Anaemia Based on Parity in Finland during 2006-10. Paediatr Perinat Epidemiol. 2014 Sep; 28(5):372-80.

[7] Liu X, Zhang W Effect of maternal age on pregnancy: A retrospective cohort study. Chin MedJ (Eng1).2014; 127(12):2241-6

[8] Dimetry SR, El-Tokhy HM, Abdo NM, Ebrahim MA, Eissa M. Urinary tract infection \& adverse outcome of pregnancy Effect of maternal age on pregnancy: A retrospective cohort study. J Egypt Public health Assoc. 2007; 82 (3-4): 203-18.

[9] Abeysena C,Jayawardana P,de A Seneviratne R. Maternal haemoglobin level at booking visit and its effect on adverse pregnancy outcome. AustNZJObstetGynaecol. 2010Oct;50 (5):423-7.

[10] Gunawansa N, Randeniya C ,Clinical Obstetrics for Undergraduates, Rhesus Isoimmunization, March 2001 